\title{
Weighted Factors for Opinion Credibility's in Twitter
}

\author{
M. Thandar and S. Usanavasin
}

\begin{abstract}
In sentiment analysing, most researches focus on only opinion classification and summarization of opinion results. However when we classify sentiment information, which information can identify whether this opinion is credible or not? Do we believe result of this topic can be positive if positive results come from unreliable source and negative results come from reliable source in sentiment analysing? For this reason, we propose a method for calculating of opinion credibility based on authors' expert knowledge. In our method we have two components: sentiment analyzer and opinion credibility calculator. We use two machine learning techniques: Naïve Bayes (NB) and Support Vector Machine (SVM) for sentiment analyzer. To define expert knowledge, we use author's profile, list features and author's tweets behaviour for weight factors in twitter. In opinion calculator, we produce opinion credibility value combine with sentiment analyzer result and expert knowledge score. Thereafter we converse all of our defined author's expert weight factors to analyses which weight factors weightier than others and which factors can support more credible when we compute opinion credibility. To evaluate our method we used weighted kappa statics for accuracy of opinion credibility.
\end{abstract}

Keywords - conversion weight, expert knowledge, machine learning, opinion credibility, sentiment analyzing, twitter, weight factors

\section{INTRODUCTION}

Explosion of social media is a way of connecting people with each other and also fast growing with effects on individual behaviour. Sentiment analysis is a main point of social media research including Twitter, Facebook, blogs, and user forums. Nowadays most of the sentiment analysing focus on opinion classification and summarization the opinions result. For example L. G. Moya et al., [13] address the aspect-based summarization task for retrieving product features from a collection of free text customer reviews about a product or service. M. Hu and B. Liu [6] generate feature-based summaries (FBS) of customer reviews of product sold online. We concern how we can verify sentiment results when positive results come from unreliable source and negative results come from reliable source. Therefore we propose a method to assess credibility of opinions expressed in tweets. We assume it can be

Manuscript received Dec. 5, 2015. This research was partially supported by the Center of Excellence in Intelligent Informatics, Speech and Language Technology and Service Innovation (CILS) and by NRU grant at SIIT, Thammasat University.

M. Thandar is with the School of Information Computer and communication Technology (ICT), Sirindhorn International Institute of Technology (SIIT), Thammasat University, Thailand.

S. Usanavasin is with SIIT, Thammasat University, Thailand. She is with the Department of Information Technology, SIIT, Thammasat University, Thailand. provided if we consider how familiar with the topic of a tweet's author for a given topic. For authors' expertise, we take into account author's profile, List features and authors' tweets behaviour in twitter. Based on these features, we produce authors' expertise six weight factors to compute opinion credibility. Moreover we also identify which weight factors are weightier than other factors. We will discuss the rest of this paper as follows: Section 2 is the literature review and related work. Section 3 follows with our method: credibility of opinions and analysing of which weight factors are weightier than others. Experiments results are presented in Section 4. We conclude the paper and discuss future work in Section 5.

\section{LITERATURE REVIEW}

We consider on sentiment analysis and author's expert knowledge to discover credibility of opinions. Most of the earlier works in sentiment analysis researches are based on results of positive or negative and they summarized results for sentiment analysis. M. Hu and B. Liu [6] examined customer opinion. They used Tree Map visualization (a method for displaying hierarchical data by using nested rectangles) to display clusters and their associated sentiment. K. Bafna and D. Toshniwal [7] deal with sentiment analysis by generating features based on summarization of customer's opinions. They used an association mining technique top fine frequent feature and used opinion lexicon and sentiword-net to identify semantic polarities for product review. B. Pang et al., [10] used three machine learning techniques: Naïve Bayes, Maximum entropy and Support Vector Machine (SVM) to classify movie review documents for sentiment analysis. A. Pak and P. Parubok [8] performed a linguistic analysis of collected corpus and determine positive, negative and neutral sentiment based on multinomial Naïve Bayes classifier that used N-gram and POS-tags as features. M. S. Neethu and R. Rajasree [9] classified tweets as positive, negative and extracted peoples' opinion about electronic products using Nave Bayes and SVM classifier. P. W. Liang and B.R. Dai [12] design a system called opinion miner which integrated machine learning techniques and domain specific data. They used unigram Naïve Bayes for extracting tweets and determined that is an opinion or not. They used Mutual Information and $\mathrm{X}^{2}$ feature selection for short text classification to discard some useless features. A. Go et al., [11] classify tweets with emoticons for distant supervised learning.

For identifying expertise in social media, A. Pal and S. Counts [3] proposed features and methods that can be used to produce a ranked list of top authors for a given topic for identifying topical authorities in microblogging environments. They found that Gaussian-based ranking is an efficient way to 
find the most authoritative users and to rank users. N. K. Sharma et al., [1] inferred a user's expertise by analysing the meta-data of Twitter Lists features. J. Weng et al., [4] measure influence of users in Twitter creating TwitterRank. It measured influence taking both topical similarity between users and the link structure into account. It calculated topical distribution of a user using Latent Dirichlet Allocation (LDA). They used PageRank algorithm to identify authorities on each topic. Y. Namihira et al., [2] proposed the topic and opinion classification based information credibility analysis system for twitter depending on user's expertise knowledge. This is similar to our approach but our approach is analysing the credibility of opinions in twitter messages.

\section{PRoposed Methodology}

In order to assess credibility of opinions, there are two parts in our approach: sentiment analyzer and opinion credibility calculator. Sentiment analyzer classifies tweets polarity and opinion credibility calculator calculates weight factors of author's expert knowledge and show credibility of tweet's polarity result. Figure 1 shows our overview system to find credibility result of tweet polarity. Before we apply our method, we make preprocessing steps: remove non-English word (using WordNet to determine whether this word is English or not), remove url, \# and @ (prefix, suffix), remove stop words, stemming, case folding.

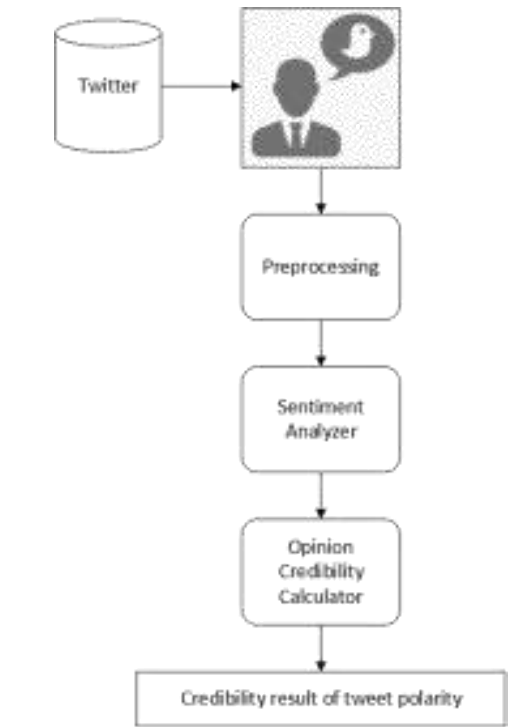

Fig. 1: Overview system of credibility result of tweet polarity

\section{A. Sentiment Analyzer}

Sentiment Analyzer classifies a given tweet as either positive or negative. In this part, we use two machine learning strategies: Naïve Bayes (NB) and Support Vector Machine (SVM) to use which one is compatible for our specific topic.

Naïve Bayes is based on Bayesian theorem and one of the most basic text classification techniques. It analyze all the features in feature vector individually and these features are independent of each other. The conditional probability theorem is shown in equation (1). $\mathrm{X}$ is defined as feature vector and $\mathrm{y}_{\mathrm{i}}$ is the class label.

$$
P\left(X \mid y_{i}\right)=\prod_{i=1}^{m} P\left(x_{i} \mid y_{i}\right)
$$

Support Vector Machine (SVM) is a useful and popular technique in classification for tweet dataset. SVM finds a linear separating hyperplane with the maximal margin in this higher dimensional space [5]. We choose Linear SVM to classify tweet polarity with Rapid Miner [16] software in our sentiment analyzer. Equation (2) means to find maximum hyperplane and separates documents from one class or other. $c_{i}$ is positive and negative with class of document $d_{i} . \alpha_{i}$ is to solve dual optimization problem.

$$
w=\sum_{i} \alpha_{i} c_{i} d_{i}, \alpha_{i} \geq 0
$$

\section{B. Opinion Credibility Calculator}

We utilize authors' expert knowledge in opinion credibility calculation for a specific topic. For author's background knowledge, we use twitter features: author's profile, List features and tweets behaviors. Author profile contains important information that indicates expertise of author, such as his/herself summarized interests, career information and links to his/her personal web page. Twitter Lists allow users to organize people they are following into labeled groups. They contain self-reported expertise indicator and follower's judgment about one's expertise and provide straightforward cues about this judgment to other users. Author tweets tweeted almost everything such as their daily activities comment on news promotion of their company, etc. N. K. Sharma et al., [1] claim that in List feature: name and description express valuable semantic cues and they are included in the lists according to their topics and expertise. Consequently we can conclude author's profile, List name and description can identify who they are in a specific topic. We select Noun and Adjective (N-gram approach \{unigram and bigram\}) using NLP toolkit [17]. Then we extract conceptual keywords that are related with a given topic using ontology concept. After that we calculate ratio of number of related keywords to total number of raw keywords for a given topic. This strategy produces first weight factor $W_{B L}$ (author expert knowledge using bio and List) to define weight of author's expert knowledge in Eq. (3).

$$
W_{B L}=\frac{\text { number of related keywords for a given topic }}{\text { total number of raw keywords }}
$$

This step is to continue computing of expert score based on author's tweets behavior and his/her network activities. We consider author's topic related ratio (TR), ratio of author's tweets retweeted by other users (RT), ratio of author's friends and followers $\left(\mathrm{F}_{1}, \mathrm{~F}_{2}\right)$ and author's opinions ratio (OP) for given topic. $\mathrm{Eq}$ (4) to $\mathrm{Eq}$ (8) represent calculation of five weight factors $\left\{\mathrm{W}_{\mathrm{TR}}\right.$ : topic related ratio, $\mathrm{W}_{\mathrm{RT}}$ : author's retweeted ratio, $\mathrm{W}_{\mathrm{F} 1}$ : author's friends ratio, $\mathrm{W}_{\mathrm{F} 2}$ : author's follower ratio, $\mathrm{W}_{\mathrm{OP}}$ : author's opinions ratio $\}$ for each author. 


$$
\begin{gathered}
W_{T R}=\frac{\text { number of author's related tweets for a given topic }}{\text { number of author's all tweet }} \\
\text { W }_{R T}=\frac{\text { number of author's tweet retweet by others for a given topic }}{\text { all } \text { author tweets for a given topic }} \\
W_{F 1}=\frac{\text { number of friends for a given topic }}{\text { total number of author's friends }} \\
W_{F 2}=\frac{\text { number of followers for a given topic }}{\text { total number of author's followers }} \\
W_{O P}=\frac{\text { number of author's negative or positive tweets }}{\text { all past opinions of author's tweets }}
\end{gathered}
$$

To achieve expert score, we combine all of these weight factors. Finally we compute expert score for a given topic by adding our six weight factors based on author's bio, list features and author's tweet behavior.

$$
\text { Expert } \text { score }=\frac{W_{B L}+W_{T R}+W_{R T}+W_{F 1}+W_{F 2}+W_{O P}}{6}
$$

When we get expert score, we combine that expert score with the result of tweet polarity from sentiment analyzer and calculate credibility tweet polarity value $C_{o p}$ (Opinion Credibility).

$$
C_{O P}=\text { tweet's polarity } \times \text { Expert score }
$$

\section{Conversion of weight factors}

In this part, we compute which weight factors are weightier than other factors when we identify credibility of opinion. Before changing weight factors in expert score, we assume all of weight factors have equal weight. Our purpose is conversion of each weight factors and determine which factors is weightier than others. We compute $C_{o p}$ (Opinion Credibility) according to their value and evaluate with using weighted kappa statics [15]. Based on conversion of weight factors value result, we identify which weight factors is more influential than other factors when we calculate credibility of opinions.

\section{EXPERIMENT RESULT}

We crawl data from Obamacare (unofficial name) that is an American Government's Healthcare policy. To classify tweets' polarity, we use 2000(training dataset) and 200(testing data).

\section{A. Experiment for Sentiment Analyzer}

Our Sentiment Analyzer produces labelling tweet's polarity (positive and negative). We use Naïve Bayes classifier and Linear SVM classifier using RapidMiner [16] tool built in function. The result are shown in Table I and II. Naïve Bayes is slightly lower accuracy than SVM and the accuracy results are $88.25 \%$ for Naïve Bayes and $90.50 \%$ for SVM.

TABLE I: RESULTS OF TRAINING CLASSIFICATION FOR NAÏVE BAYES (NB)

\begin{tabular}{|l|l|l|l|}
\hline polarity & true positive & true negative & class precision \\
\hline positive & 925 & 160 & $85.25 \%$ \\
\hline negative & 75 & 840 & $91.80 \%$ \\
\hline class recall & $92.50 \%$ & $84.00 \%$ & \\
\hline
\end{tabular}

TABLE II: RESULTS OF TRAINING CLASSIFICATION FOR SVM

\begin{tabular}{|c|c|c|c|}
\hline polarity & true positive & true negative & class precision \\
\hline positive & 920 & 110 & $89.32 \%$ \\
\hline negative & 80 & 890 & $91.75 \%$ \\
\hline class recall & $92.00 \%$ & $89.00 \%$ & \\
\hline
\end{tabular}

\section{B. Experiment for Opinion Credibility Calculator}

To evaluate accuracy of opinion credibility, we use weighted kappa $\left(\mathrm{K}_{\mathrm{w}}\right)$ statics and it measures assessment of how much rank for the agreement between our system and human raters [15]. For human raters, we use 10 volunteers who are familiar in a given topic. We set three ranges for our opinion credibility values such as: highest $(\mathrm{h}>=70 \%)$, lowest $(\mathrm{l}<=30 \%)$ and middle $(\mathrm{h}<=\mathrm{m}>=\mathrm{l})$.

$$
\begin{gathered}
K_{w}=\frac{P_{o}(w)-P_{e}(w)}{1-P_{e}(w)} \\
P_{o}(w)=\frac{1}{N} \sum_{s=1}^{R} \sum_{t=1}^{R} w_{s t} n_{s t} \\
P_{e}(w)=\frac{1}{N} \sum_{s=1}^{R} \sum_{t=1}^{R} w_{s t} r_{s} c_{t}
\end{gathered}
$$

$\mathrm{K}_{\mathrm{w}}=$ rank value of agreement between system and rater $\mathrm{P}_{\mathrm{o}}(\mathrm{w})=$ the weighted observed proportional agreement between the system and rater

$\mathrm{P}_{\mathrm{e}}(\mathrm{w})=$ the weighted proportional agreement expected just by chance is estimated

$\mathrm{N}=$ number of tweets

$\mathrm{R}=$ ranges of our credibility level

$\mathrm{r}_{\mathrm{s}}=\sum_{s=1}^{R} n_{s t}$

$\mathrm{c}_{\mathrm{t}}=\sum_{s=1}^{R} n_{s t}$

$\mathrm{n}_{\mathrm{st}}=$ the number of observations that have been classified as belonging to category( $\mathrm{s}$ ) by system and to category $(\mathrm{t})$ by rater $\mathrm{w}_{\mathrm{st}}=$ the degree of discrepancy between two categories $\mathrm{s}$ and $\mathrm{t}$

To determine value of kappa statics strength, we use J. R. Landis and G. G. Koch [14] approach. If it has 1 for maximum value and it indicates the result is perfect and 0 (zero) means poor result according to the following table III. In our evaluation, $\mathrm{R}=\{$ highest $(\mathrm{h})$, lowest (l) and middle $(\mathrm{m})\}$, $\mathrm{N}=100$ for each positive and negative. Table IV and V show number of agreement between our system and human rater for each positive and negative. For the matrix w follows as:

$$
w=\left(\begin{array}{lll}
1 & 1 & 0 \\
1 & 1 & 1 \\
0 & 1 & 1
\end{array}\right)
$$

TABLE III: LEVEL OF AGREEMENT MEASURE FOR KAPPA STATICS $\left(\mathrm{K}_{\mathrm{W}}\right)$

\begin{tabular}{|c|c|}
\hline Kappa Statistics $(\mathrm{Kw})$ & Strength of Agreement \\
\hline$<0.000$ & Poor \\
\hline $0.000-0.20$ & Slight \\
\hline $0.21-0.40$ & Fair \\
\hline $0.41-0.60$ & Moderate \\
\hline $0.61-0.80$ & Substantial \\
\hline $0.81-1.00$ & Almost Perfect \\
\hline
\end{tabular}


TABLE IV: Number OF AGREEMENT BeTWEen System AND RATER For POSITIVE RESULT

\begin{tabular}{|c|c|c|c|c|}
\hline \multirow{2}{*}{ System } & \multicolumn{4}{|c|}{ Rater } \\
\cline { 2 - 5 } & $\mathrm{h}$ & $\mathrm{m}$ & $\mathrm{l}$ & Total \\
\hline $\mathrm{h}$ & 18 & 7 & 3 & 28 \\
\hline $\mathrm{m}$ & 6 & 9 & 11 & 26 \\
\hline $\mathrm{l}$ & 8 & 6 & 32 & 46 \\
\hline Total & 32 & 22 & 46 & 100 \\
\hline
\end{tabular}

TABLE V: Number OF AGREEMENT BETWEEN SySTEM AND RATER For NEGATIVE RESULT

\begin{tabular}{|c|c|c|c|c|}
\hline \multirow{2}{*}{ System } & \multicolumn{4}{|c|}{ Rater } \\
\cline { 2 - 5 } & $\mathrm{h}$ & $\mathrm{m}$ & 1 & Total \\
\hline $\mathrm{h}$ & 18 & 7 & 3 & 28 \\
\hline $\mathrm{m}$ & 6 & 9 & 11 & 26 \\
\hline $\mathrm{l}$ & 8 & 6 & 32 & 46 \\
\hline Total & 32 & 22 & 46 & 100 \\
\hline
\end{tabular}

After we compute evaluation for no weight, we get result \{ positive: 0.60 (Moderate), negative: 0.52 (Moderate) in Table VI.

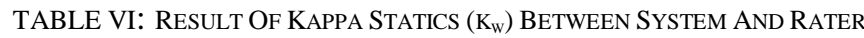
FOR NO WEIGHT

\begin{tabular}{|c|c|}
\hline \multicolumn{2}{|c|}{ Result of Kappa Statistics } \\
\hline Positive & Negative \\
\hline 0.60 (Moderate) & 0.52 (Moderate) \\
\hline
\end{tabular}

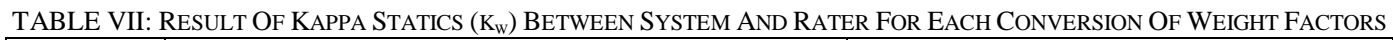

\begin{tabular}{|c|c|c|c|c|c|c|c|c|}
\hline & \multicolumn{7}{|c|}{ Weight Factors } & \multicolumn{2}{c|}{ Result of Kappa Statistics (Kw) } \\
\hline & $\mathrm{W}_{\mathrm{BL}}$ & $\mathrm{W}_{\mathrm{TR}}$ & $\mathrm{W}_{\mathrm{RT}}$ & $\mathrm{W}_{\mathrm{F} 1}$ & $\mathrm{~W}_{\mathrm{F} 2}$ & $\mathrm{~W}_{\mathrm{OP}}$ & Positive & Negative \\
\hline No weight & 1 & 1 & 1 & 1 & 1 & 1 & 0.60 (Moderate) & 0.52 (Moderate) \\
\hline \multirow{5}{*}{ Step 1 } & 0 & 1 & 0 & 0 & 0 & 0 & 0.53 (Moderate) & 0.49 (Moderate) \\
\cline { 2 - 10 } & 1 & 0 & 0 & 0 & 0 & 0 & 0.42 (Moderate) & 0.44 (Moderate) \\
\cline { 2 - 10 } & 0 & 0 & 1 & 0 & 0 & 0 & 0.37 (Fair) & 0.21 (Fair) \\
\cline { 2 - 10 } & 0 & 0 & 0 & 0 & 1 & 0 & 0.34 (Fair) & 0.24 (Fair) \\
\cline { 2 - 10 } & 0 & 0 & 0 & 1 & 0 & 0 & 0.18 (Slight) & 0.17 (Slight) \\
\hline \multirow{5}{*}{ Step 2 } & 0.5 & 0 & 0 & 0 & 0 & 1 & 0.07 (Slight) & 0.08 (Slight) \\
\hline \multirow{5}{*}{ Step 3 } & 0.4 & 0.4 & 0.1 & 0 & 0.1 & 0 & 0.47 (Moderate) & 0.46 (Moderate) \\
\cline { 2 - 10 } & 0.3 & 0.3 & 0.2 & 0 & 0.2 & 0 & 0.38 (Fair) & 0.34 (Fair) \\
\cline { 2 - 10 } & 0.2 & 0.2 & 0.3 & 0 & 0.3 & 0 & 0.19 (Slight) & 0.17 (Slight) \\
\cline { 2 - 10 } & 0.1 & 0.1 & 0.4 & 0 & 0.4 & 0 & 0.09 (Slight) & 0.11 (Slight) \\
\cline { 2 - 10 } & 0 & 0 & 0.5 & 0 & 0.5 & 0 & 0.3 (Moderate) \\
\hline \multirow{5}{*}{ Step 4 } & 0.4 & 0.4 & 0 & 0.1 & 0 & 0.1 & 0.17 (Slight) & 0.15 (Slight) \\
\cline { 2 - 9 } & 0.3 & 0.3 & 0 & 0.2 & 0 & 0.2 & 0.15 (Slight) & 0.12 (Slight) \\
\cline { 2 - 9 } & 0.2 & 0.2 & 0 & 0.3 & 0 & 0.3 & 0.09 (Slight) & 0.08 (Slight) \\
\cline { 2 - 9 } & 0.1 & 0.1 & 0 & 0.4 & 0 & 0.4 & 0.08 (Slight) & 0.07 (Slight) \\
\hline \multirow{5}{*}{} & 0 & 0 & 0 & 0.5 & 0 & 0.5 & 0.05 (Slight) & 0.07 (Slight) \\
\hline
\end{tabular}

\section{Experiment for conversion of weight factors}

We also evaluate conversion of our six weight factors values to identify the most weighted factors in our defined weight factors.

\section{Step 1: Set one factor to ' 1 ' and the rest to ' 0 ':}

Firstly, we set each of weight factor stage to " 0 ", expect one factor and give that factor stage to "1". E.g., IF $\left(W_{B L}\right.$ (stage) $=1$ $\left(W_{T R}=W_{R T}=W_{F 1}=W_{F 2}=W_{O P}=0\right)$ THEN $C_{O P}=$ ? In this step, we find $\mathrm{W}_{\mathrm{BL}}$ and $\mathrm{W}_{\mathrm{TR}}$ are the first highest weight factors, $\mathrm{W}_{\mathrm{RT}}$ and $\mathrm{W}_{\mathrm{F} 2}$ is the second highest weight factors and $\mathrm{W}_{\mathrm{F} 1}$ and $\mathrm{W}_{\mathrm{OP}}$ are the lowest weight factors among these six weight factors in Table VII: Step 1.

Step 2: Set two highest weight factors' value to 0.5 and the rest to 0
Based on the step 1 experiment result, we notice $\mathrm{W}_{\mathrm{BL}}$ and $\mathrm{W}_{\mathrm{TR}}$ are the first highest weight factors in our weight factors. Therefore we give these two highest weight factors to $\left\{\mathrm{W}_{\mathrm{BL}}\right.$ $=0.5, \mathrm{~W}_{\mathrm{TR}}=0.5 \mathrm{f}$ and set 0.0 for the rest and calculate accuracy of our opinion credibility result again in Table VII: Step 2.

Step 3: Decrease first two highest weight factors' value $\left(W_{\mathrm{BL}}, \mathbf{W}_{\mathrm{TR}}\right)$ and increase second two highest weight factors' value $\left(W_{\mathrm{RT}}, \mathrm{W}_{\mathrm{F} 2}\right)$

In this step, we decrease two highest weight factors' values $\left(\mathrm{W}_{\mathrm{BL}}\right.$ and $\left.\mathrm{W}_{\mathrm{TR}}\right)$ from 0.5 to 0 by 0.1 . Simultaneously, we also increase the second two highest weight factors' values $\left(\mathrm{W}_{\mathrm{RT}}\right.$, $\mathrm{W}_{\mathrm{F} 2}$ ) from 0 to 0.5 by 0.1 . The results are in the following Table VII: Step 3.

Step 4: Decrease first two highest weight factors' value $\left(\mathbf{W}_{\mathrm{BL}}, \mathbf{W}_{\mathrm{TR}}\right)$ and increase two lowest weight factors' value $\left(\mathbf{W}_{\mathrm{F} 1}, \mathbf{W}_{\mathrm{OP}}\right)$ 
In step 4, we decrease two highest weight factors' values $\left(\mathrm{W}_{\mathrm{BL}}\right.$ and $\mathrm{W}_{\mathrm{TR}}$ ) from 0.5 to 0 by 0.1 . Simultaneously, we also increase two lowest weight factors' values $\left(\mathrm{W}_{\mathrm{F} 1}, \mathrm{~W}_{\mathrm{OP}}\right)$ from 0 to 0.5 by 0.1. The results are in the following Table VII: Step 4.

Based on our conversion of weight factors value, we find that $\mathrm{W}_{\mathrm{BL}}$ and $\mathrm{W}_{\mathrm{TR}}$ are the most important weight factors in our six factors because we discover our accuracy result values are decreased when we reduce their values $\left(\mathrm{W}_{\mathrm{BL}}\right.$ and $\left.\mathrm{W}_{\mathrm{TR}}\right)$.

\section{CONCLUSION}

We develop a new method for credibility of opinion based on expert knowledge. When we measure opinion credibility, we use six weight factors for expert knowledge. We use weighted kappa statistics and get the accuracy result for our opinion credibility: positive $\{0.60$ (Moderate) $\}$ and negative $\{0.52$ (Moderate) \}. Moreover we converse our weight factors, we find some factors are important and give more value for opinion credibility result. Based on our result, $\mathrm{W}_{\mathrm{BL}}$ (author description and list feature) and $\mathrm{W}_{\mathrm{TR}}$ (author topic ratio) are weightier than other factors. For future, we will focus to modify our expert score calculation based on conversion of our weight factors result and compare with different topic.

\section{REFERENCES}

[1] N. K. Sharma, S. Ghosh, K. Gummadi, "Inferring Who-is-Who in the Twitter Social Network," In: Proceeding of the 2012 ACM workshop on Workshop on online social networks, pp. 55-60. ACM Press, New York (2012). http://dx.doi.org/10.1145/2342549.2342563

[2] Y. Namihira, N. Segawa, K. Kawai, "High Precision Credibility Analysis of Information on Twitter," International Conference on Signal-Image Technology \& Internet-Based Systems, pp. 909-915. Kyoto (2013). http://dx.doi.org/10.1109/SITIS.2013.148

[3] A. Pal, S. Counts, "Identifying topical authorities in microblogs," In Proceedings of the fourth ACM international conference on Web search and data mining. ACM Press, New York, pp. 45-54. ACM Press, New York (2011) http://dx.doi.org/10.1145/1935826.1935843

[4] J. Weng, E. Lim, J. Jiang, "TwitterRank: finding topic-sensitive influential twitterers," In: Proceedings of the third ACM international conference on Web search and data mining, pp. 261-270. ACM Press, New York (2010). http://dx.doi.org/10.1145/1718487.1718520

[5] C. Hsu, C. Chang, C. Lin, "A practical guide to support vector classification," Dept. Comput.Sci., National Taiwan Univ., Taipei, Taiwan,2010.[Online].Available:http://www.csie.ntu.edu.tw/ cjlin/papers/ guide/guide.pdf.

[6] M. Hu, B. Liu, "Mining and summarizing customer reviews," In: Proceedings of the tenth ACM SIGKDD international conference on Knowledge discovery and data mining, pp. 168-177. ACM Press, New York (2004) http://dx.doi.org/10.1145/1014052.1014073

[7] K. Bafna, D. Toshniwal, "Feature Based Summarization of Customers' Reviews of Online Products," 17th International Conference in Knowledge Based and Intelligent Information and Engineering Systems, vol. 22, pp. 142-151, 2013. http://dx.doi.org/10.1016/j.procs.2013.09.090

[8] A. Pak, P. Parubok, "Twitter as a Corpus for Sentiment Analysis and Opinion Mining," In: Proceedings of the Seventh conference on International Language Resources and Evaluation (2010)

[9] M. S. Neethu, R. Rajasree, "Sentiment analysis in twitter using machine learning techniques," Fourth International Conference on Computing, Communications and Networking Technologies, pp. 1-5 (2013) http://dx.doi.org/10.1109/iccent.2013.6726818

[10] B. Pang, L. Lee, S. Vaithyanathan, "Thumbs up? Sentiment Classification using Machine Learning Technique," In: Proceedings of the ACL-02 conference on Empirical methods in natural language processing (2002).
[11] A. Go, R. Bhayani, L. Huang, “ Twitter sentiment classification using distant supervision", CS224N Project Report, Stanford (2009)

[12] P. W. Liang, B.R. Dai, "Opinion Mining on Social Media Data," Mobile Data Management (MDM), IEEE 14th International Conference, pp. 91-96, IEEE Press, Milan (2013) http://dx.doi.org/10.1109/mdm.2013.73

[13] L. G. Moya, H, A. Sanchez, R. B. Llavori, "Retrieving Product Features and Opinions from Customer Reviews," R. Intelligent Systems, IEEE (Volume: 28, Issue: 3 ), 2013

[14] J. R. Landis, G. G. Koch , "The measurement of observer agreement for categorical data," Biometric, vol.33, pp.159-174, 1977 http://dx.doi.org/10.2307/2529310

[15] J. Cohen, "Weighted kappa: Nominal scale agreement provision for scaled disagreement or partial credit," Psychological bulletin, vol. 70(4), pp.213-220, 1968. http://dx.doi.org/10.1037/h0026256

[16] www.rapidminer.org

[17] www.nltk.org

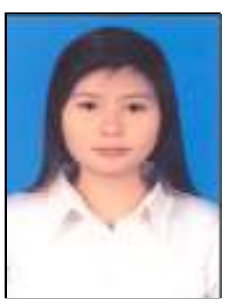

Mya Thandar was born in 1990, Yangon, Myanmar. She graduated from University of Computer Science (UCSY), Yangon, Myanmar, with bachelor degree in computer science in 2009.

She is currently working on Master of Computer Engineering in School of Information Computer and communication Technology (ICT), Sirindhorn International Institute of Technology (SIIT), Thammasat University Thailand.

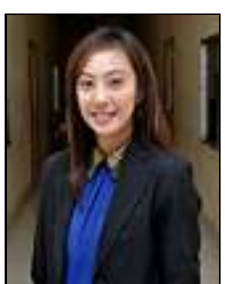

Dr.Sasiporn Usanavasin received her Bachelor of Science in Information Technology from Sirindhorn International Institute of Technology (SIIT) at Thammasat University in Thailand. She received her Master's and $\mathrm{PhD}$. degrees from Keio University in Japan. Currently she is a lecturer at Sirindhorn International Institute of Technology (SIIT), Thammasat University, Thailand. Her research interests are in Software Engineering, Service Science and Knowledge Engineering fields. 